\title{
El crecimiento impulsado por el mercado interno como respuesta a la crisis en américa latina. ¿Una utopía movilizadora?
}

Domestica market-led growth as a response to the crisis in Latin America. A mobilising utopia?

\section{Pierre Salama}

Economista, Profesor de la Facultad de Economía-Universidad de París XIII (Villetaneuse).

salamapierrealbert@yahoo.fr

http://pero.wanadoo.fr/pierre.salama/

Este artículo desarrolla temas expuestos por el autor en la conferencia que dictó en la Universidad Nacional de Lanús (UNLa) el día jueves 12 mayo de 2011. Traducción del francés a cargo de Emilio Taddei, docente-investigador del Departamento de Planificación y Políticas Públicas de la Universidad Nacional de Lanús (UNLa).

\section{Fecha de recepción:}

10 de junio de 2011

Fecha de aceptación:

15 de julio de 2011

\section{Resumen}

El objetivo de este artículo es indagar si una estrategia centrada en una distribución más justa de los ingresos y la consiguiente expansión del mercado interno puede contribuir a un incremento sostenido del crecimiento económico en América Latina. Aunque los obstáculos a tal estrategia parecen convertirla en una mera utopía, se argumenta que ella parece estar materializándose en algunos países de la región como Brasil y Argentina. La primera parte del artículo repasa los efectos de la crisis internacional actual en el tejido industrial de las principales economías latinoamericanas, evaluándose en la segunda las posibilidades de éxito de una apuesta por el mercado interno, teniendo en cuenta la gravitación de dos factores: la amplitud de la apertura externa y el peso de la desigualdad en la distribución del ingreso.

Palabras clave: crisis - mercado interno, - política económica - América Latina.

\section{Abstract}

The aim of this article is to examine whether a strategy centred on a fairer income distribution and the resulting expansion of the domestic market may contribute to a sustained increase in the economic growth in Latin America. Although obstacles to that strategy may look like mere utopia, it is argued that it appears to be materializing in some countries of the region such as Brazil and Argentina. 
The first part of his article discusses the effects of the $\mathrm{cu}$ rrent international crisis on the industrial web of the main Latin American economies whereas the second part focuses on the possibilities of success from a bet on the domestic market, taking into account the influence of two factors: the scope of the external opening and the significant role of inequality in income distribution.

Key words: crisis - domestic market - economic policy Latin America - income distribution.

Luego de décadas de fuerte crecimiento, la crisis de los años ochenta sacudió profundamente a América Latina. El nivel del PBI (producto bruto interno) que se había alcanzado en 1980 sólo logró equipararse catorce años después, al igual que la tasa de pobreza que se ubicó en los mismos valores de 1980 al cabo de veinticinco años (Jiménez, 2010). En un continente marcado por fuertes desigualdades y, salvo raras excepciones, por políticas fiscales regresivas, por mecanismos de transferencia social bastante modestos en comparación con los vigentes en Europa, por una liberalización del comercio acotada (con la excepción de México y de algunos países de América Central), la crisis internacional de 2008 interrumpió una fase de crecimiento relativamente importante iniciada en 2003-2004.

A diferencia de lo que había sucedido durante las crisis de los años ochenta, la crisis de 2008 está fundamentalmente asociada a los países en desarrollo, antes que a las dificultades propias de la región. En este sentido se asemeja a la crisis de los años treinta. También a diferencia de las crisis de los años ochenta la de 2008, si bien es aguda, pareciera por el momento tener una duración relativamente acotada ya que desde fines de 2009 asistimos a un proceso de relanzamiento relativamente ágil del crecimiento económico. Conviene sin embargo ser prudente y no confundir un momento del ciclo con una temporalidad más larga del mismo, sobre todo porque se trata de una crisis estructural. En el estadio actual de la crisis internacional y en la medida en que aún no fue redefinida la arquitectura financiera internacional conviene ante todo ser prudente (y por qué no también un poco escépticos) respecto a la solidez y durabilidad de este crecimiento. Se trata entonces de una retomada del crecimiento económico aún frágil pero al igual que sucedió durante las crisis de la década de los ochenta y sobre todo durante los años treinta del siglo pasado la experiencia de esta crisis se pone de manifiesto en la mutación de las estructuras productivas que, si bien aún es dificil descifrar con precisión, modifica las estructuras de la gobernabilidad.

El objetivo de este artículo es indagar si luego de años de una apertura creciente un nuevo régimen de crecimiento centrado en una distribución más justa de los ingre- 
sos, con la consecuente expansión del mercado interno que ello supone, es capaz de contribuir a un incremento durable del crecimiento económico. Frente a la profunda desigualdad imperante y a los poderosos conflictos de intereses que impiden tanto una reforma fiscal como una política redistributiva más onerosa para los sectores sociales más pudientes de las sociedades latinoamericanas, la apuesta por el mercado interno luego de la prioridad otorgada al mercado externo y legitimada en los años noventa por las recomendaciones y políticas del Consenso de Washington - pareciera presentar un carácter aparentemente utópico. Sin embargo es necesario constatar que en algunos países como Brasil y en menor medida la Argentina esta "utopía" parece estar materializándose de manera incipiente. Las políticas contracíclicas adoptadas luego del estallido de la crisis internacional son diferentes a las implementadas en los años anteriores que, como señalamos, estaban inspiradas en el Consenso de Washington y se caracterizaban por la reducción de los gastos sociales practicados el calor de las crisis cambiarias y que precipitaron los ciclos recesivos. Las nuevas políticas inspiradas en un "keynesianismo pragmático" buscan promover el sostenimiento de la demanda y conducen a una disminución de los excedentes primarios. Al menos hasta inicios de 2010 estas medidas atenuaron el costo social de la crisis en lugar de acentuarlo.

Al menos en un aspecto estas políticas contracíclicas se inscriben en una relativa continuidad con las que fueron adoptadas en algunos países a inicios de la década pasada: una débil disminución de las desigualdades, una política social más sostenida y una retomada del crecimiento. ¿Podemos entonces considerar que la crisis internacional acelera de forma subterránea un proceso ya iniciado? O bien de forma más pesimista ¿podemos pensar que solo se trata de balbuceos y que una vez que se confirme la tendencia al crecimiento el modelo excluyente anterior, con algunos "retoques", volverá a prevalecer? ¿El retorno del mercado interno, utopía movilizadora, o el retorno al modelo precedente, más abierto y menos vulnerable pero más frágil? (Salama, 2009a, 2009b). En la primera parte repasaremos los efectos de la crisis sobre el tejido industrial de las principales economías latinoamericanas y en la segunda parte intentaremos evaluar las posibilidades de éxito de la "apuesta por el mercado interno" privilegiando dos factores: la amplitud de la apertura externa y el peso que guarda la desigualdad de los ingresos.

\section{De una crisis a otra}

\subsection{Las inesperadas consecuencias de la crisis de 1930: un modelo original de industrialización en Argentina, Brasil y México.}

La industrialización de los países periféricos se inscribe en una división internacional del trabajo que reposa sobre la explotación de productos primarios por parte de las potencias dominantes y hace más compleja sus formaciones sociales, al mismo tiempo que constituye una amenaza para los empleos de las empresas de esos mismos países. Por otra parte la industrialización en la periferia abre la puerta a potenciales procesos de 
interpelación a las lógicas de dominación que los países dominantes ejercen en el plano internacional. Esto explica la hostilidad manifiesta de estos países a la industrialización y el débil desarrollo de los mercados internos en los países periféricos. Esta relación de dominación se alteró significativamente en primer lugar como consecuencia de la primera guerra mundial y luego por la gran crisis de los años treinta. Los cambios ocurridos al final de la segunda guerra mundial marcados por la consagración de la hegemonía internacional de los Estados Unidos, que reemplazó el lugar ocupado hasta entonces por Gran Bretaña ya debilitada y en declive, crearon un contexto más favorable para la industrialización de los países de la periferia.

La crisis de los años treinta tuvo dimensiones mayúsculas. Sus repercusiones sobre las economías en desarrollo fueron en general considerables. Con el deterioro de los términos de intercambio y la caída pronunciada del volumen de exportaciones de productos primarios la capacidad de importación de estos países se vio afectada considerablemente. La duración de la crisis, no prevista por los políticos, tuvo consecuencias diferenciadas según los países. Algunos no sufrieron cambios profundos y continuaron insertándose de forma tradicional en la división internacional del trabajo. Otros, menos numerosos, luego de una fase de crisis más o menos prolongada experimentaron una "industrialización no pensada", según la feliz expresión de la CEPAL (Comisión Económica para América Latina de las Naciones Unidas). Esta industrialización no planificada fue el resultado de un conjunto de condiciones: a) existencia de un tejido industrial mínimo generado por la propia actividad exportadora (por ejemplo talleres de reparación de máquinas a vapor utilizadas en la actividad del transporte de materias primas, economías de aglomeración ligadas a la edificación de puertos y ciudades, y otras); b) demanda proveniente de la difusión más o menos importante de relaciones mercantiles o asalariadas según la naturaleza de las exportaciones; c) apoyo efectivo a los ingresos de los exportadores a través de la compra en moneda local de una parte de su producción. El respeto de estas condiciones y la prolongación imprevista de las restricciones externas condujo a un modelo original de industrialización: un círculo virtuoso de sustitución de importaciones de bienes livianos que permitió un fuerte crecimiento en un contexto internacional en crisis y que creó consecuentemente un mercado interno.

La dimensión del mercado interno aumentó a medida que se desarrolló el proceso de sustitución de importaciones. La inversión funcionó creando empleos ya que los bienes producidos eran de baja intensidad de capital. La provisión de mano de obra y de trabajadores provino de los flujos migratorios internacionales por un lado y por otro, y de forma creciente, de la migración interna del campo a las ciudades. La contratación de estos trabajadores se tradujo en un incremento del proceso de monetización ya que como campesinos se encontraban poco monetizados. La creciente monetización de la economía reemplazó y amplió la demanda de los bienes exportables valorizando así la producción destinada al mercado interno que se encontraba en vías de constitución. El 
círculo es virtuoso en este sentido. La dinámica del crecimiento proviene entonces del aumento de la demanda pero a diferencia de un proceso keynesiano clásico, este incremento está más ligado a la monetización de la fuerza de trabajo que al aumento de los salarios. Este aspecto esencial del proceso de sustitución de importaciones livianas de los años 1930-1940 es a menudo ignorado por los economistas.

La crisis estructural de los años treinta dio nacimiento a un nuevo régimen de crecimiento "impulsado desde adentro", según la expresión de la CEPAL. En este sentido la crisis dio lugar a un proceso de restructuración y de superación donde "lo viejo que no termina de morir (la economía exportadora) cede lugar a lo nuevo que intenta nacer (la industrialización por sustitución de importaciones)".

La crisis de los años treinta que estimuló la "industrialización no pensada" en algunos países de la periferia condujo a la aparición de regímenes políticos particulares y específicos. La expansión de la industria modificó la formación social y poco a poco emergieron nuevos conflictos de intereses entre las clases sociales y al interior de estas. Estos conflictos se exacerbaron cuando la sustitución de importaciones livianas se debilitó. Por un lado, y como consecuencia de la prolongación de la crisis internacional, la capacidad de importación no aumentó y por otro la estructura de estas importaciones se hizo cada vez más rígida. Así la posibilidad de producir bienes de equipamiento y productos intermedios que no podían importarse en cantidad suficiente se hizo crecientemente dificil. Esto por dos razones. Por un lado porque los bienes que debían producirse eran más intensivos en capital y la inversión requerida era mayor; por otro porque no existían bolsas de valores susceptibles de centralizar los capitales que no podían ser garantizados por pequeños empresarios individuales. El estado emerge entonces como único agente capaz de invertir en dichos sectores. Pero no resulta de esto que el estado invierte porque objetivamente deba hacerlo. Todo depende de la configuración de los conflictos sociales y de la manera de superarlos y darles sentido. En Argentina, Brasil y México la existencia de los regímenes políticos de carácter "cesarista" (Perón, Vargas, Cárdenas) habría de permitir pasar efectivamente de un crecimiento estimulado por la sustitución de importaciones livianas a un crecimiento estimulado por la sustitución de importaciones pesadas gracias a la intervención directa del estado como inversor en estos sectores. En cierta medida el estado substituye al empresario ausente como resultado del tamaño insuficiente del mercado interno existente (Mathias y Salama, 1983).

La industria pesada y los sectores energéticos y de infraestructura van a desarrollarse si las condiciones políticas lo permiten. Esto posibilita superar durante algún tiempo los obstáculos casi infranqueables producidos por el modelo de crecimiento precedente. La crisis de los años treinta tuvo por lo tanto efectos muy importantes en Argentina, Brasil y México tanto desde el punto de vista económico como político.¿Tiene la crisis de 2008 la capacidad de producir efectos de una amplitud semejante? 


\subsection{La crisis de 2008}

La crisis que se inicia en 2008 es diferente de la de los años treinta. Es distinta en sus causas ya que la financierización económica prevalece sobre otro tipo de dinámicas. Las consecuencias de la financierización sobre el debilitamiento de los ingresos del trabajo y el endeudamiento de los hogares en algunos países desarrollados son hoy conocidas. No podemos sin embargo clasificar la crisis de 2008 como una crisis de realización argumentando que durante un período de tiempo prolongado los salarios aumentaron poco en los países desarrollados debido al hecho que el endeudamiento estimuló un tipo de demanda que hubiera sido débil si éste no hubiera existido. Tampoco puede explicarse como una crisis de sobreacumulación ya que en general la tasa de inversión sigue siendo bastante modesta en los países desarrollados y las capacidades productivas disponibles son débiles.

La crisis de 2008 es la crisis de los efectos de la globalización comercial y financiera sobre los salarios y la inversión productiva. La globalización comercial y los condicionantes externos vinculados a los bajos niveles salariales existentes en los países asiáticos incitan a la "desconexión" de la evolución salarial en relación con la evolución de la productividad, no solamente en los países desarrollados sino también en las economías emergentes latinoamericanas. La globalización financiera estimula una reorganización de las empresas para aumentar su rentabilidad inmediata. La distribución entre salario y ganancia se realiza en perjuicio del primero, con excepción de los salarios de los dirigentes empresariales y bancarios y de los beneficios financieros que aumentan considerablemente. Se produce entonces un doble efecto sobre los salarios: limitaciones externas, limitaciones financieras en la distribución del valor agregado y un efecto virtuoso provisorio gracias a la invención de nuevos productos financieros titularizados que resultan muy atractivos.

Si esta crisis es de orden estructural, como creemos, no es una reactivación coyuntural la que habrá de permitir superar las causas que la produjeron. Solamente una reforma profunda de la arquitectura financiera internacional y la imposición de nuevas reglas que regulen el comercio internacional -y que tengan en cuenta las condiciones éticas y ambientales de la producción- permitirán superar la crisis de 2008. A la luz de lo que sucedió durante la gran crisis de los años treinta con la aparición de un nuevo modelo de industrialización y la reactivación del crecimiento en los grandes países de América Latina, podemos considerar que la crisis de 2008 puede quizás permitir la emergencia de posibilidades para un nuevo ciclo de crecimiento en los países emergentes latinoamericanos, aunque es preciso ser prudentes respecto a la duración y significación del mismo. ¿Se trata de una fase alcista de un ciclo tendencialmente descendente? ¿O bien estamos frente al inicio de un ciclo de desacople más durable? Es dificil responder a estas preguntas ya que las respuestas dependen, como en los años treinta, de las reacciones políticas frente a los conflictos de intereses en juego, más particularmente frente a los conflictos distributivos. 
La apertura más o menos pronunciada de las economías latinoamericanas a los intercambios comerciales y a los flujos financieros estableció canales de transmisión que favorecieron los efectos de contagio entre los países desarrollados y entre estos y los países en vías de desarrollo (OMC-OCDE, 2009; OCDE, 2009; FMI, 2009) ${ }^{1}$. La crisis de 2008 fue severa (de cinco a diez puntos menos de crecimiento dependiendo de cada caso particular en las distintas economías latinoamericanas emergentes) (Salama, 2009a y 2009b) y fue seguida en forma bastante rápida por una reactivación del crecimiento. El relanzamiento de la dinámica que conocieron estos países reposa esencialmente en un cierto dinamismo de sus mercados internos y en una oferta sostenida de las exportaciones hacia las economías asiáticas (China y en menor medida India). Sin embargo si nuevamente sobreviniera una crisis en los países desarrollados -como parece expresarlo la amenazadora crisis sobre las deudas soberanas de algunos países europeos- el dinamismo de las economías latinoamericanas podría verse afectado. Los efectos sobre las economías emergentes podrían ser más o menos devastadores de acuerdo al grado de apertura comercial de las mismas, al tipo de bienes exportados, a la intensidad de la globalización financiera y a la estructura de los stocks/flujos de capital extranjero (bonos, acciones, inversiones directas) ${ }^{2}$, a la magnitud de sus reservas reales netas ${ }^{3}$.Y esto no solamente porque habría una caída de la demanda externa sino también porque en algunas economías emergentes la posibilidad de que se consolide la dinámica del mercado interno depende del lapso de tiempo que separa a ambas crisis. Cuanto más desarrollado es el mercado interno mayores son las posibilidades de resistir a una crisis externa.

En el marco de nuestro artículo no podemos considerar esta eventualidad, que nos parece poco probable en un futuro cercano. La hipótesis que planteamos es que los países desarrollados mantendrán un crecimiento débil en la medida en que no se implementen las reformas estructurales. Es en este contexto de crecimiento económico débil en los países industrializados y de potenciales turbulencias financieras que sugerimos la posible aparición de nuevos regímenes de crecimiento económico centrados en la dinamización de los mercados internos en las economías latinoamericanas.

\section{La historia puede repetirse?}

En los años veinte la participación de las exportaciones de las grandes economías latinoamericanas en las exportaciones mundiales era más importante que la alcanzada en nuestros días luego de treinta años de apertura continua. Comúnmente se caracteriza el período de los años ochenta como el momento de la liberalización de los mercados y se piensa por lo tanto que las economías estaban más globalizadas que hoy. Este razonamiento olvida sin embargo que el mundo de esa época difería del de hoy en relación a un punto esencial: el grado de monetización. En las economías desarrolladas el autoconsumo en las zonas rurales, donde residía la mayor parte de la población, era importante. En las economías de América Latina era aún mayor. La relación de las exportaciones con el PBI tiene por lo tanto una significación reducida, ya que remite a magnitudes 
monetizadas. Pero resulta que una parte importante de la reproducción de los individuos pasaba por circuitos no monetizados y por lo tanto no considerados en el cálculo del PBI. Hoy la monetización es casi total y esta relación es más pertinente que en la década de 1920. Hoy el mercado interno existe, no hay que construirlo. La única forma de incrementarlo es aumentando los ingresos del trabajo y los beneficios sociales lo cual, en el contexto de la globalización, solo puede realizarse si paralelamente se mantiene la competitividad relativa del país. Si esto no sucede las importaciones sustituyen a la producción interna. A diferencia de los años treinta, el crecimiento centrado en la expansión del mercado interno supone respetar los desafios que plantea la competitividad, a menos que se reimplanten medidas proteccionistas. Estas pueden estar legitimadas por argumentos que refieren al respeto de las condiciones éticas y ambientales de producción o por el estímulo al "compre nacional" como puede ya observarse. La apuesta en favor del mercado interno debe entonces ser analizada a partir de este doble contexto de globalización y monetización casi completa, ausente en los años treintas y considerando dos variables: la contribución al crecimiento por parte del comercio exterior y la amplitud de las desigualdades.

\subsection{Contribución mayor del comercio exterior, aunque relativamente débil, al crecimiento.}

La contribución del comercio exterior al crecimiento económico puede analizarse desde dos perspectivas: una estrictamente contable y la otra basada en mecanismos económicos que contemplan los efectos de arrastre.

Desde el punto de vista contable la evaluación se centra en las exportaciones y en las importaciones, es decir las exportaciones netas de las importaciones. Aún cuando un país está abierto a la economía mundial las primeras contribuyen positivamente a la tasa de crecimiento de dicho país cuando su balanza comercial es negativa, y viceversa. El caso de los países asiáticos, y particularmente el de China, es interesante ya que a menudo se presentan como ejemplo cuando se destacan los efectos benéficos sobre el crecimiento que tiene la expansión de las exportaciones. Cuando se considera el período 2000-2008 la contribución media de las exportaciones netas se eleva a 10,2\% de la tasa de crecimiento en China. Esto significa que para una tasa de crecimiento medio del PBI de $10,2 \%$ esta contribución es solamente de 1,1 puntos mientras que la de inversión es de 5 puntos y el consumo total de 4,1 puntos. Es cierto que en el transcurso de este período crece con el aumento de los excedentes comerciales de China: según Goldstein y Xie $(2009)^{4}$ la contribución de las exportaciones netas pasó de 5\% entre 2001 y 2004 a más de $20 \%$ entre 2005 y 2007.

Contribución contable y contribución económica no son lo mismo. Aún cuando la contribución contable al crecimiento por parte las exportaciones netas sea débil, o inclusive negativa, la contribución económica puede ser importante. Las exportaciones 
pueden jugar un papel importante en el crecimiento. Inversamente pueden no tener ninguna influencia sobre el crecimiento si la tasa de apertura es importante. Tomemos dos ejemplos de economías fuertemente abiertas: el caso de México y el de Corea. En el primer caso el crecimiento no es estimulado desde el exterior, en el segundo sí.

En México el saldo de la balanza comercial continúa siendo deficitario, contrariamente a lo que sucede en Brasil y Argentina estos últimos años. La estructura de las exportaciones de México se compone entre 10\% y15\% de exportaciones de petróleo, cuyo precio es volátil. El resto se divide casi por mitades entre la exportación de productos destinados principalmente al mercado interno mexicano y la otra mitad de productos destinados casi exclusivamente a los mercados externos (casi exclusivamente a Estados Unidos). El fuerte aumento de la tasa de apertura durante estos últimos treinta años se explica por la difusión de las exportaciones de productos manufacturados ensamblados producidos en las "maquiladoras". Su valor agregado es bajo y generan poco efecto multiplicador (Palma, 2005). La contribución económica al crecimiento es por lo tanto reducida. El crecimiento del PBI resulta bajo mientras que el de las exportaciones es significativo. El efecto multiplicador es débil (Ibarra, 2008).

En el caso coreano el crecimiento es estimulado por las exportaciones pero la relación es más compleja de lo que generalmente se piensa. Según los trabajos de D. Rodrik (1995) y contrariamente a lo postulado por la doxa liberal no fueron ni el auge de las exportaciones ni la apertura indiscriminada de la economía los que permitieron la aceleración del crecimiento en los años sesentas. Las importaciones se componen sobre todo de productos intermedios y de bienes de equipamiento sofisticados. El fuerte incremento de las mismas refleja el de las inversiones. Son entonces las inversiones las que estimulan el crecimiento. Estas inversiones se destinan en gran medida a la producción de bienes de exportación, que son fuente de divisas. La relación es entonces la siguiente: aumento de la tasa de inversión, crecimiento de las importaciones, crecimiento de las exportaciones. Solo después de esto el saldo de la balanza comercial resulta positivo. Analizada desde un punto de vista económico la contribución positiva al crecimiento de las exportaciones, pero también de las importaciones de bienes de equipamiento sofisticados - fuente de mayor eficacia de las inversiones -, se explica por la política industrial seguida por el gobierno tendiente a favorecer la producción local de insumos necesarios para la fabricación de los productos exportados. Esta política apunta fundamentalmente a incrementar el valor agregado producido localmente y explica a su vez el aumento de las inversiones y de las importaciones de bienes de equipamiento. La contribución de las exportaciones al crecimiento económico proviene de una intensificación del tejido industrial que la acompaña a través de un aumento directo de las inversiones en el sector que produce bienes destinados a la exportación y un alza indirecta de los sectores que producen insumos para estos productos. Desde un punto de vista keynesiano el efecto multiplicador del aumento de las inversiones prevalece 
sobre el efecto negativo de las importaciones y se suma al de las exportaciones. Esto que acabamos de referir en relación al caso coreano se aplica también para un buen número de economías asiáticas. Dinamizar el mercado interno por medio de una redistribución de ingresos no supone minimizar o despreciar el papel desempeñado por el mercado externo. No se trata de un "juego de suma cero".

La relación "mercado externo-mercado interno" es por lo tanto más compleja de lo que parece a primera vista. Como dicen los filósofos, estamos en presencia de dos datos "separados pero dependientes". Este punto de vista es poco considerado por los economistas.

Dicho esto es preciso subrayar que en América Latina durante las dos últimas décadas la apertura comercial fue moderada y la contribución de las exportaciones al crecimiento económico fue modesta. Contrariamente a lo que podríamos imaginar las economías emergentes latinoamericanas no conocieron una dinámica importante de apertura comercial. Con excepción de México y de algunos pequeños países de América Central; Brasil, Argentina y un buen número de países de la región mantuvieron globalmente su participación en las exportaciones mundiales ${ }^{5}$. Dado que estas crecieron en promedio durante este período dos veces más rápido que el PBI mundial, la globalización se acentuó pero a un ritmo más o menos equivalente al promedio mundial. A pesar del aumento sustancial del nivel de apertura de Brasil entre 1990 (11,7\%) y 2004 (26,9\%) el peso de este país en el comercio internacional sigue siendo aún marginal y relativamente estable entre 1975 (1,1\%) y 2005 (1,1\%) (Kliass y Salama, 2008); inclusive si posteriormente creció levemente en virtud del significativo aumento de las materias primas. Inversamente, el crecimiento de las exportaciones de China es mucho más rápido que la media mundial. Si en 1975 la participación del país asiático en el comercio internacional era equivalente a la de Brasil $(0,9 \%)$ luego experimentó un crecimiento inocultable: $1,9 \%$ en $1990,3,9 \%$ en $2000,7,4 \%$ en 2005 y llega a 8,9\% en 2009 (Instituto de Estudos para o Desenvolvimento Industrial y OMC-OCDE). La globalización comercial es en este caso más rápida e importante que en el caso de Brasil. Este último país y la Argentina se abrieron a la economía mundial pero aún no son economías tan abiertas como a menudo se señala.

En estos países, y contrariamente a lo que se observó en los países asiáticos, el nivel de financierización fue y es elevado, más incluso que en los países asiáticos. Sin embargo en estos casos la apertura financiera no se acompañó, o poco, con el desarrollo de productos financieros titularizados de alto riesgo en los balances bancarios, aunque estos últimos se vieron afectados por falta de liquidez como consecuencia de las repatriaciones de capitales que se produjeron en el inicio de la crisis y no por la necesidad de "limpiar" sus balances. Ello no obstante y aunque sus balances estuvieron poco afectados por productos financieros de alto riesgo, el comportamiento de los bancos privados se adaptó 
al observado en los países desarrollados: los créditos a la economía disminuyeron. El financiamiento de las inversiones y de las exportaciones se hizo crecientemente dificil. Los créditos a la economía no se desplomaron ya que los gobiernos facilitaron el acceso al crédito e impusieron bajas tasas de interés (pluralidad de las tasas subvencionadas para la compra de automóviles, de viviendas, a la exportación, etc.) a través de las bancos públicos. Esta política se vio favorecida por la existencia de grandes bancos públicos, como el Banco Nacional de Desarrollo (BNDES) en Brasil y, en sentido contrario, se hizo más difícil ante la inexistencia o debilitamiento de los mismos.

\subsection{Fuertes desigualdades, obstáculos a una reactivación del crecimiento a través del mercado interno.}

Las desigualdades de ingresos son particularmente elevadas en la gran mayoría de los países latinoamericanos (Salama, 2006), aunque en varios países disminuyeron levemente entre 2002 y 2008 (CEPAL 2009; López-Calva y Lustig 2009; Salvadori Dedecca, 2010).

Esta evolución es importante. Sus causas son múltiples: funcionamiento diferenciado del mercado de trabajo, transferencias sociales más importantes, política fiscal menos regresiva, disminución demográfica y aumento de la tasa de empleo femenino. La contribución de estos dos últimos factores a la disminución de las desigualdades es modesta según López-Calva y Lustig (2009:40 y ss.). En Brasil el número de adultos por hogar explica 6,6\% de la reducción de las desigualdades entre 2000 y 2006. En Argentina esta cifra es de 8\% y en México de 10,3\%. Lo esencial de esta disminución de las desigualdades proviene de una mejora de las condiciones de trabajo (empleo, salario) y relativamente poco del aumento de los ingresos que no provienen del trabajo ${ }^{6}(26 \%$ en Argentina, 15,1\% en México). Más precisamente en Brasil vemos que la mejoría en los ingresos del trabajo es más importante en los sectores de bajos ingresos que en los sectores de altos ingresos. La razón entre los ingresos del trabajo del 5\% más rico de la población y los del 50\% más modesto pasa de 14,3 en 1993 a 13,5 en 2008 y finalmente la razón entre el 5\% de los más ricos y el 25\% de los más pobres varió de 23,6 a 18,6 (Salvatori Dedecca, 2010: 16). Estas cifras pueden parecer sorprendentes ${ }^{7}$. En parte se explican por el fuerte aumento del salario mínimo y, con ello, por el monto de las jubilaciones pagadas por el sector público ${ }^{8}$. Obedecen también por otra parte al crecimiento del empleo y a la evolución de la estructura de empleos (Salama, 2007). Pero lo más importante aquí es que contrariamente a lo que sostiene la doxa las transferencias sociales gravitan poco en la evolución de las desigualdades. Veremos esto con mayor detalle.

Los trabajos de la OCDE (2008), los de Goni, Humberto-López y Serven (2008) muestran claramente la débil influencia de las transferencias sobre el nivel de concentración de los ingresos medido en base al coeficiente de Gini. Cuando consideramos la diferencia entre ingreso bruto (que incluye los aportes sociales) y el "ingreso de mercado" 
se observa que el impacto de estas transferencias en los niveles de concentración de ingresos es alto en Europa pero muy débil en América Latina. Si consideramos el ingreso disponible (con aportes e impuestos directos) y el ingreso bruto (con aportes) se observa que el impacto de los impuestos en la disminución de las desigualdades es mucho más importante en Europa que en América Latina ${ }^{10}$. Esta es la razón por la cual según la OCDE (2008:130 y siguientes) la diferencia entre el coeficiente de Gini de los "ingresos de mercado" y los ingresos netos de las transferencias y de los impuestos directos en 2006 es del orden de dos puntos en América Latina contra veinte puntos en Europa. La conclusión es simple: mientras que los niveles de concentración de ingresos anteriores a las transferencias y a los impuestos directos solo presentan entre cuatro y cinco puntos de diferencia máxima entre los países de América Latina y los países europeos, estas diferencias son mucho más importantes cuando se consideran las transferencias netas de impuestos (del orden de 20-25 puntos). En promedio el coeficiente de Gini en América Latina pasa de 51,6 a 49,6 mientras que en Europa oscila entre 47,6 a 28,2.

A partir de estos datos podemos comprender que bajo la influencia de los trabajos de Celso Furtado muchos economistas hayan visto en estos niveles de desigualdad y en el bajo nivel de gastos sociales la razón de la tendencia al estancamiento económico (Salama 2006, primer capítulo) y que, por el contrario, la ligera disminución de las desigualdades y el aumento de los gastos sociales hayan podido dinamizar el crecimiento en la década del 2000 e insuflar dinamismo al mercado interno.

\section{Conclusión}

Resulta difícil realizar hipótesis sobre el futuro de los países latinoamericanos sin distinguir las diferentes trayectorias en el transcurso de la última década o década y media. Brasil, Argentina y México tienen características comunes. Entre las más visibles podemos destacar la existencia de fuertes desigualdades en los ingresos, más importante en Brasil que en Argentina; la leve reducción de estas desigualdades; una apertura al comercio internacional aún modesta a excepción de México; el peso que cobró una parte de las exportaciones primarias en relación al total de las exportaciones en Argentina y en Brasil; la dificultad para exportar productos industriales sofisticados, menor en Brasil que en México o en Argentina y por último la tendencia a la apreciación de la tasa de cambio real con excepción de Argentina en la década pasada.También tienen recorridos diferentes. Brasil y México experimentaron en la última década una tasa modesta de crecimiento medio de su PBI. En 2004 la tasa de crecimiento aumentó en Brasil y la Argentina experimentó un crecimiento de tipo asiático. En Argentina la caída del PBI fue muy elevada en 2009, pero aún así menor que la registrada en México. En la década pasada en Brasil las desigualdades disminuyeron más visiblemente que en Argentina y México y los gastos sociales aumentaron proporcionalmente al PBI en Brasil y en Argentina, mientras que se mantuvieron estables en México (Alfonso y Dain op. cit.). 
Estas tendencias, al igual que la aceleración del crecimiento experimentada a partir de 2003-2004 nos conducen a preguntarnos si aún antes del estallido de la crisis de 2008 no estaríamos en presencia de un nuevo régimen de crecimiento en Brasil y en Argentina (aunque en este último caso esta tendencia sería más moderada). Este régimen parece estar estimulado por el aumento de la demanda interna y, si bien aún se vislumbra "en filigrana", su percepción se ve eclipsada por el efecto provocado por el aumento de las exportaciones de productos primarios resultante del aumento de los precios internacionales de estos productos.

Estos países se encuentran en una encrucijada. La reactivación económica y las políticas contracíclicas $^{11}$, adoptadas con posterioridad al estallido de la crisis internacional, podrían servir de trampolín para la definición de un nuevo régimen de crecimiento. A través de la profundización de lo que tímidamente se expresó en los años 2000 estos países podrían de esta forma "aprovechar" la crisis internacional para disminuir sensiblemente las desigualdades de ingresos y estimular el papel desempeñado por el mercado interno en el crecimiento económico, de forma similar a lo que ocurrió en los años 1933-1934 con la gran crisis de esa década.

El regreso masivo de capitales sobre los llamados mercados financieros emergentes en América Latina, el crecimiento del PBI y las dificultades para sostener la demanda en la medida en que esta podría incrementar los costos del trabajo refuerzan el peso político de aquellos que desearían “cerrar el paréntesis abierto por la crisis” y regresar al régimen de crecimiento excluyente de los años anteriores. Esta tentación es mayor en la medida en que el regreso de los capitales se traduce en una nueva tendencia a la apreciación de las monedas nacionales luego de su caída en 2008-2009 y resulta favorable a los inversiones extranjeras, pero no favorece a los exportadores de productos industriales (disminución de la competitividad debida al aumento del costo salarial expresado en dólares que no siempre compensa la disminución del valor de sus importaciones de productos intermedios y de bienes de equipamiento). Podemos pensar que México será el país más tentado a seguir por ese camino ya que su comercio exterior está dirigido casi exclusivamente a los Estados Unidos y Canadá y los intereses extranjeros son muy fuertes en el sistema bancario del país azteca. En sentido contrario un nuevo ciclo de la crisis internacional podría favorecer la búsqueda de una política anticíclica que favoreciera la demanda, estimulando la búsqueda de otros socios comerciales y permitiendo una nueva depreciación del peso que pudiera compensar el aumento del costo del trabajo.

El mantenimiento de la política de apoyo a la demanda interna tiene más posibilidades de materializarse en Brasil y en la Argentina. Pero encuentra sin embargo múltiples limitaciones. La dimensión absoluta de la población de Brasil (aún más que en Argentina) y la existencia del Mercosur le permiten disponer de un mercado interno suficientemente amplio para una serie de productos desde el punto de vista de la valorización 
del capital, pero parece insuficiente para insuflar un crecimiento durable estimulado por el mercado interno, en la medida en que el peso de las finanzas y sus efectos sobre la distribución de los ingresos no puedan ser contenidos. Por más deseable que sea el incremento de los salarios más bajos este es aún insuficiente en función de la amplitud de la miseria existente. El obstáculo de la política fiscal regresiva debe ser superado, lo cual necesariamente generará importantes conflictos de intereses que ya están presentes y pueden percibirse desde hace algunos años.

En los años treinta el mercado se creó a través de la monetización "a marcha forzada" producida por la industrialización y la impulsó. Hoy el mercado interno no puede contribuir en forma durable al crecimiento si no se pone en práctica un verdadero Welfare State capaz de compensar la atonía de la demanda externa con productos industriales. No apostar a esta vía es aceptar pasivamente el regreso a una especialización internacional centrada en la producción de productos primarios bajo el pretexto de aprovechar el dinamismo que hoy tiene la demanda internacional. Es elegir el camino más fácil pero también el de la fragilidad del futuro. Es optar por un regreso a los años anteriores a la década del treinta... extraño regreso de la Historia.

\footnotetext{
${ }^{1}$ Es necesario recordar que de manera general en las economías emergentes los bancos tenían pocos activos de riesgo elevado y si al inicio de la crisis hubo una sequía de liquidez, ella fue sobre todo porque los capitales "fugaron" de esos países para alimentar la liquidez de las casas matrices de las empresas y de los bancos multinacionales y de los inversores internacionales. Esta es la razón de la caída de las monedas respecto al dólar. El regreso de los capitales atraídos por la alta rentabilidad de las bolsas emergentes no condujo sin embargo a un incremento significativo de los préstamos. Es por ello que los bancos públicos tuvieron que funcionar como posta de los bancos privados en quiebra.
}

${ }^{2}$ El contagio es más o menos consecuente según los países y la amplitud de sus respectivas globalizaciones. Algunos indicadores sintéticos permiten evaluarlo. Es lo que intentan hacer con mayor o menor éxito las instituciones internacionales (FMI, 2009), ya que según un indicador construido por la OCDE $(2009,42)$ México sufriría menos la crisis que otros países. Sin embargo esto fue justamente lo que no sucedió.

${ }^{3}$ Se trata de un punto importante y poco analizado. Las reservas se forman ya sea con excedentes de la balanza comercial, con excedentes de la balanza de capitales, o con ambos. Solamente las primeras son reservas reales, mientras que las segundas son muy sensibles a la coyuntura y pueden verse amputadas por un retorno parcial de los capitales, como fue el caso a finales de 2008. Sobre este punto ver Bradesco (24/12/2009).

${ }^{4}$ Vale señalar como ejemplo que la contribución neta de Corea del Sur en el período es más elevada $(28,6 \%)$ al igual que la de Singapur (27,3\%). La de Alemania fue de 64\% (balanza comercial fuertemente excedentaria) y la de los Estados Unidos de - 4,3\% (balanza comercial deficitaria).

${ }^{5}$ Además de las críticas formuladas por Birdsall y Hamoudi (2002) a los trabajos de Dollar y Kray (2000) subrayamos que este indicador está lejos de ser perfecto para las economías cuyas exportaciones están constituidas en gran parte o exclusivamente por productos primarios como en el caso de los países menos avan- 
zados (PMA). En efecto la volatilidad de los mercados de materias primas es particularmente pronunciada. El numerador de esta razón se encuentra entonces muy afectado por la evolución de los precios de las materias primas y por lo tanto también el propio indicador. Definir o no las economías como "globalizantes" de acuerdo a la evolución de esa razón y pretender establecer una relación con la tasa de crecimiento del PBI es poco pertinente desde un punto de vista científico.

${ }^{6}$ Esta categoría es muy homogénea. Remite tanto a las transferencias sociales, a los alquileres como también a los ingresos de capital.

${ }^{7}$ Dos observaciones: los ingresos del 1\% crecen mucho más rápido como en el conjunto de los países occidentales; los ingresos del capital (intereses, dividendos) están muy deficientemente registrados.

${ }^{8}$ En muchos países se puede observar una relación entre el monto de las jubilaciones pagadas y el salario mínimo; el incremento relativo de éste aumenta el monto de las jubilaciones. De forma más general las políticas sociales, de acuerdo a la definición que de las mismas hacen los latinoamericanos (educación y de transferencias sociales, y, fundamentalmente la salud y las jubilaciones) son relativamente débiles aunque están en aumento en algunos países como Brasil. Según un trabajo de Afonso y Dain (2009) en el período 1985 1990 solamente en tres países (Argentina, Chile y Costa Rica) los gastos sociales excedían 13\% del PBI. En el período 2006-2007 son nueve países los que se encuentran en esta situación, entre ellos Brasil (24,4\% del $\mathrm{PBI}$ ), pero no Chile. En el primer período señalado los gastos sociales en México eran inferiores a 9\% del PBI pero aumentaron en el segundo período a $11,2 \%$ del PBI.

${ }^{9}$ Según esta definición el ingreso de mercado no incluye las contribuciones sociales, a diferencia del ingreso bruto que sí las incluye. El ingreso de mercado es por lo tanto el pago que el asalariado recibe de su empleador. El ingreso disponible es el ingreso que dispone el asalariado luego de la deducción de los impuestos directos.

${ }^{10}$ Esta diferencia sería aún mayor si hubiéramos podido incluir en este trabajo los impuestos indirectos, más importantes en América Latina que en Europa (CEPAL 2009: Gómez Sabaini et al. 2008). Los impuestos indirectos son en general más regresivos que los impuestos directos ya que los paga el conjunto de los ciudadanos mientras que no ocurre lo mismo con los impuestos directos.

${ }^{11}$ Políticas contracíclicas que se caracterizan fundamentalmente por el estímulo a la demanda interna, tanto en el orden fiscal (reducción de ciertos impuestos) como en el monetario (tasa de interés artificialmente baja para la compra o la venta de ciertos productos) y en el plano presupuestario (efectos debidos al aumento del salario mínimo, de las jubilaciones, de las transferencias sociales) y no así o en menor medida por políticas de infraestructura pública materializadas en grandes emprendimientos como en el caso de China (Khatiwada, Jiménez 2009). 


\section{Referencias Bibliográficas}

Afonso, J. R. y Dain, S. (2009) Dos décadas de la descentralización del gasto social en América Latina: una evaluación preliminar. Santiago de Chile: CEPAL, documento de trabajo no publicado.

Alvarez Estrada, Daniel (2009) “Tributación directa en América Latina: equidad y desafios. Estudio del caso de México”. CEPAL: Serie Macroeconomía del desarrollo No. 91 (agosto).

Birdsall, N. y Hamoudi, A. (2002) Commodity dependence, trade and growth : when "openness" is not enough. Washington Center for Global Development. Working Paper $\mathrm{n}^{\circ} 7$ (May).

Bradesco (2009) "Em que medida o elevado deficit externo brasileiro em 2010 sera capaz de conter a apreciaçao do real?" Destaque Depec, n¹04, 28 de diciembre.

CEPAL (2009) Balance preliminar de las economias de America Latina y el Caribe. Santiago de Chile.

Dollar, D. y Kray, A. (2000) Growth is good for the poor. Washington DC: World Bank www.worldbank.org/research.

FMI (2009) World economic Outlook : Crisis and Recovery. IMF: Washington D.C.

Galindo A., Izquierdo, A. y Rojas-Suarez, L. (2010) Financial Integration and Foreign Banks in Latin America, How they impact the transmission of external financial shocks. IDB Working Paper $n^{\circ} 116$

Goldstein, M. y Xie, D. (2009) The Impact of the Financial Crisis on Emerging Asia. Washington D.C.: Peterson Institute for International Economics, Working paper $n^{\circ} 9 / 11$. Gomez Sabaini, J. y Rossignolo, D. (2008) Argentina: análisis de la situación tributaria $y$ propuestas de reformas impositivas destinadas a mejorar la distribución del ingreso. www. estimacionestributarias.com/archivos/sabaini\%202008.pdf

Goni, E., Lopez, Humberto, J. E. y Serven, L. (2008) Fiscal Redistribution and Income Inequality in Latin America. Policy Research Working Paper, ${ }^{\circ} 4487$

Ibarra C.(2008) "La paradoja del crecimento lento de México", Revista de la Cepal 95:85-102.

IEDI (2006) Carta Iedi Brasilia

Jimenez, J. P. (2010) Crisis, recuperación y espacios de política: desafíos de la política fiscal. Santiago de Chile: CEPAL, Documento de trabajo.

Khatiwada, S. (2009) Stimulus Packages to Counter Global Economic Crisis, Geneva, International Institute for Labor Studies, Discussion Paper DP/196.

Kliass, P. y Salama, P. (2008) 'La globalisation au Brésil: ¿responsable ou bouc émissaire?" Lusotopie 38.

Lopez-Calva, L. F. y Lustig, N.( 2009) The recent decline of Inequality in Latin America: Argentina, Brazil, Mexico and Peru. ECINEQ, Working Paper $\mathrm{n}^{\circ} 140$.

Mathias, G. y Salama, P. (1983) L'Etat sur développé. Paris : Maspéro-La découverte.

OCDE (2008) Perspectives économiques de l'Amérique latine 2009. Bruxelles: OCDE. 
OCDE (2009) Regional Economic Outlook, Western Hemisphere. Bruxelles: OCDE.

OMC-OCDE (2009) Globalisation and Emerging Economics. Paris: OCDE.

Palma, G. (2005) "The seven main "stylised facts" of the Mexican economy since trade liberalization and Nafta". Journal of Industrial and Corporate Change 14 (6):941-991.

Rodrik, D. (1995) "Growth policy, getting interventions right : how South Korea and Taiwan grew rich”. Economic Policy No. 20. www.nber.org/papers/w4964.pdf

Salama, P. (2006) Le défi des inégalités, une comparaison économique Amérique latine/Asie. Paris : La Découverte.

Salama, P. (2007) 'Pauvreté, le bout du tunnel?”, Problèmes d'Amérique latine, n66/67. Existe versión en castellano disponible en: http://pierre.salama.pagesperso-orange.fr/ Salama (2009a) "Argentine, Brésil, Mexique face à la crise", Revue Tiers Monde n 197. Existe versión en castellano Salama, Pierre (2009) Argentina, Brasil y México frente a la crisis internacional (Buenos Aires: Herramienta). Disponible en: http://pierre.salama. pagesperso-orange.fr/

Salama (2009b) "Forces et faiblesses de l'Argentine, du Brésil, du Mexique", in Les Suds dans la crise" (sous la direction de Ph.Hugon et P.Salama). Paris : Armand Colin. .

Salvadori Dedecca, C. (2010) “As desigualdades na sociedade brasileira”. Documento no publicado.

Titelman, D., Perez-Caldentey. E. y Pineda, R. (2009) ¿Cómo algo tan pequeño terminó siendo tan grande? Crisis financiera, mecanismo de contagio y efectos en America Latina. Revista de la CEPAL 98:7-34. 